\section{Estimating the vertex of converging lines:}

\section{Angle misperception?*}

\author{
DANIEL J. WEINTRAUB†, University of Michigan, Ann Arbor, Michigan 48104 \\ and \\ VEIJO VIRSU, University of Helsinki, Helsinki, Finland
}

The angle between two converging but nonintersecting line segments and the orientation of the display were varied; $O$ was asked to determine the vertex. A descriptive model based on four additive factors was devised to explain the systematic errors: (1) perceptual tilt of individual line segments toward the more closely aligned axis of the visual field, either horizontal or vertical, (2) tendency to place the intersection too high in the visual field, (3) orientation-dependent component of misperception of the angle between the line segments, and (4) orientation-independent component of angle misperception. A regression analysis showed that each of the four components was highly significant statistically; together, they accounted for $89 \%$ of the variance of the mean errors.

Considerable psychophysical evidence is available to lead one to the conclusion that acute angles are overestimated. A brief discussion can be found in the introduction to our previous report (Weintraub \& Virsu, 1971). Recent neurophysiological evidence (Burns \& Pritchard, 1971) was interpreted as showing that, for the cat, an acute angle is represented neurally as too large. A relatively unexploited psychophysical approach with high face validity might contribute converging evidence. Let an $O$ be asked to place $a$ dot at the apparent intersection of two line segments. If he places the dot too far away from the ends of the converging segments, then a strong inference is that the angle subtended by the segments is being underestimated. By similar reasoning, vertex placement too close is evidence for angle overestimation. Unfortunately, Os place the point of apparent intersection consistently off to the left or right of the imaginary line bisecting the angle if such errors are permitted, and what began as a more straightforward question becomes complicated in light of the data.

Our angle-estimation technique has

*Supported by a U.S. Public Health Service Research Scientist Development Award (K2-MH-35,253) to D.J.W. and by Kamarineuvos H. Rosenberg's travel grant from the University of Helsinki to V.V. Research funds were provided by National Science Foundation Grant GB 8181 and by the University of Michigan's Biomedical Sciences Institutional Support Grant PHS FR 07050.03. We thank Lillian Tong for serving as E. We also thank three perspicacious critics-two anonymous reviewers and Irwin Pollack-whose incisive comments on earliex drafts were invaluable.

tAddress: Human Performance Center. Perry Building, 330 Packard Rd., University of Michigan, Ann Arbor, Michigan $\mathbf{4 8 1 0 4}$. definite methodological advantages. Two-dimensional freedom for $O$ in determining the vertex permits maximum freedom to err, improving the opportunities to detect important sources of variation. There is no comparison stimulus required so that misperceiving the comparison is not a problem. The dot marking the vertex is removed from the immediate vicinity of the segments themselves, reducing the possibility of interaction between the dot, which marks the judgment, and the segments, which are the features to be judged. Since the lines do not form an intersection, theories concerned with retinal blurredness in the region of an intersection (Chiang, 1968) should not apply.

In the prior study (Weintraub \& Virsu, 1971), the two-dimensionality of the errors compelled us to propose that a salient component of the converging-line anomaly is the perceptual tilt of each line segment toward the more closely aligned visual axis, either horizontal or vertical, relative to the 0 . (As yet, $O$ himself has not been tilted; only displays have been tilted relative to 0 .) The proposition is not novel and is supported by other evidence (cf. Obonai, 1931; Bouma \& Andriessen, 1968; Weintraub \& Krantz, 1971). However, the principle of assimilation toward a cardinal viewing axis applies individually to each line segment of a pair comprising an angle and not to the angle subtended. It is logically possible that the outcome of the dot-setting task can be predicted by adding the tilt-assimilation errors of each line. However, the previous study (Weintraub \& Virsu, 1971) showed that when the tilt bias for a single line at a specified inclination in the visual field was computed, the inclination of the other line of the pair was not irrelevant. Angle subtended between lines is the logical construct for characterizing the interaction. Thus, the hope at the outset of the present study was to gather sufficient parametric data to make it possible to remove, via regression analysis, the tilt-assimilation component of individual lines, and then to attribute the remaining systematic bias to angle misperception.

The previously published study had divided the orientation of displays into one of two classes: symmetrical, with the angle bisector vertical in O's visual field; asymmetrical, with the more clockwise line of each subtended angle remaining vertical. For asymmetrical displays, the orientation of the bisector of a subtended angle was confounded with the size of the subtended angle. The new design manipulated orientation and size of subtended angle independently. Based upon the previous data, three subtended angles were chosen: an acute angle $(20 \mathrm{deg})$ and an obtuse angle (120 deg) that showed large errors, and one acute angle (60 deg) that induced very little error. Inclination of individual line segments was deemed to be the most critical variable, and 13 orientations of each subtended angle were selected with particular attention devoted to positions where single lines from different angles would match in inclination.

It must be emphasized that we are not wedded to regression analysis. Rather, the data led us there by virtue of uninterpretable bivariate dot settings. The resulting model (equation) is not to be viewed as the culmination of a mathematical pasttime, namely, trying to account for maximum variance with the fewest factors. The experiment was designed to measure and remove the previously identified tilt-assimilation factor in order to examine the residual as angle misestimation. This stepwise analysis revealed a second and unanticipated systematic bias: 0 seemed to place the dot representing his judged vertex too high in his visual field in addition to whatever else he did. After endeavoring to establish the existence of the second factor by argument and by gathering additional data, the factor became a post hoc addition to the model. The ensuing analysis of the residual bias revealed that it was unworthy of the title "pure" angle misperception.

The remainder of the article attempts to argue convincingly that: (1) misestimation of a subtended angle must be a function of the orientation of the angle as well as of the size of 
Table 1

Means of Judements in Millimeters: The Angle Bisector is the Ordinate $(\Psi)$ With the True Intercetion as Origin

\begin{tabular}{|c|c|c|c|c|c|c|c|c|c|c|c|c|c|}
\hline & \multicolumn{13}{|c|}{ Orientation of the Angle Bisector (Degrees) } \\
\hline & -90 & -80 & -60 & -45 & -30 & -10 & $\mathbf{0}$ & +10 & +30 & +45 & +60 & +80 & +90 \\
\hline$\frac{\overline{\mathbf{X}}}{\overline{\mathbf{Y}}}$ & $\begin{array}{r}-.06 \\
-1.42\end{array}$ & $\begin{array}{r}-.38 \\
1.18\end{array}$ & $\begin{array}{r}.79 \\
-2.69\end{array}$ & $\begin{array}{r}-.26 \\
.75\end{array}$ & $\begin{array}{r}{ }^{S} \\
-2.34 \\
1.84\end{array}$ & $\begin{array}{c}\text { btended } \\
-35 \\
5.19\end{array}$ & $\begin{array}{c}\text { Angle } 20 \\
-.05 \\
6.39\end{array}$ & $\begin{array}{r}\text { Deg } \\
.83 \\
5.70\end{array}$ & $\begin{array}{l}1.18 \\
3.52\end{array}$ & $\begin{array}{r}.34 \\
2.07\end{array}$ & $\begin{array}{r}-1.16 \\
3.81\end{array}$ & $\begin{array}{r}-.69 \\
8.27\end{array}$ & $\begin{array}{r}.18 \\
5.40\end{array}$ \\
\hline$\frac{\overline{\mathbf{X}}}{\overline{\mathbf{Y}}}$ & $\begin{array}{r}.66 \\
-3.50\end{array}$ & $\begin{array}{r}.20 \\
-3.13\end{array}$ & $\begin{array}{l}-1.23 \\
-2.63\end{array}$ & $\begin{array}{l}-1.07 \\
-4.04\end{array}$ & $-1.03^{s}$ & $\begin{array}{c}\text { btended } \\
.12 \\
.69\end{array}$ & $\begin{array}{c}\text { Angle } 60 \\
-.07 \\
1.02\end{array}$ & $\begin{array}{r}\text { Deg } \\
-.37 \\
1.27\end{array}$ & $\begin{array}{r}-.46 \\
-1.63\end{array}$ & $\begin{array}{r}-.04 \\
-1.74\end{array}$ & $\begin{array}{r}.17 \\
-.72\end{array}$ & $\begin{array}{r}-.05 \\
1.17\end{array}$ & $\begin{array}{r}-.12 \\
.92\end{array}$ \\
\hline$\frac{\bar{X}}{\mathbf{Y}}$ & $\begin{array}{r}1.39 \\
-6.70\end{array}$ & $\begin{array}{r}1.20 \\
-5.54\end{array}$ & $\begin{array}{r}2.17 \\
-2.52\end{array}$ & $\begin{array}{r}1.36 \\
-.51\end{array}$ & $\begin{array}{c}{ }^{S} \\
-.28\end{array}$ & $\begin{array}{r}\text { tended } \\
1.03 \\
-2.21\end{array}$ & $\begin{array}{c}\text { Angle } 120 \\
.18 \\
-3.36\end{array}$ & $\begin{array}{r}\text { Deg } \\
.07 \\
-2.65\end{array}$ & $\begin{array}{l}-.78 \\
-.44\end{array}$ & $\begin{array}{l}-.41 \\
-.71\end{array}$ & $\begin{array}{r}.90 \\
-1.54\end{array}$ & $\begin{array}{r}.38 \\
-4.35\end{array}$ & $\begin{array}{r}.22 \\
-6.01\end{array}$ \\
\hline
\end{tabular}

Note-Positive values of $\bar{X}$ signify that the mean was located off the angle bisector toward the more counterclockwise line of the pair forming the subtended angle. Positive values of $\bar{Y}$ signify that the mean was too far from the line segments to coincide with the true intersection.

the angle; (2) the first two factors in the analytic model are more than intervening variables, that is, more than mere terms in an equation. They are not the polynomials in $x$ of a trend analysis. Rather, they were chosen partly on the basis of outside evidence, and emerged after months of worrying the data to death. We believe that these two factors are relevant to other paradigms and other phenomena.

\section{METHOD}

The experiment is an elaboration of the earlier study (Weintraub \& Virsu, 1971), and procedural details are identical if not discussed below. The paid $O s$ were 48 students at the University of Michigan. Two converging line segments, each $30 \mathrm{~mm}$ long, .25 mm wide, were drawn with black India ink on $21.6 \times 27.9 \mathrm{~cm}$ ( $81 / 2 \times 11$ in.) rectangular sheets of white cardboard. The distance between the true point of intersection and the near ends of the line segments was always $60 \mathrm{~mm}$. With the eye-to-display distance of approximately $54 \mathrm{~cm}$ used in the experiment, $30 \mathrm{~mm}$ is equal to $3.2 \mathrm{deg}$ of visual angle.

The angle subtended between the line segments was 20,60 , or 120 deg. The subtended angles were presented randomly in 13 different orientations throughout the left-hand half of the visual field. (To be more precise, the angle bisector was never located to the right of the vertex.) The O's line of sight always coincided with the true vertex. The orientations are designated by the direction of the vertex end of the angle bisector: $-90 \mathrm{deg}$ (vertex toward the bottom), $-80,-60,-45$, $-30,-10,0 \mathrm{deg}$ (vertex toward the right), and $+10,+30,+45,+60,+80$, +90 deg (vertex toward the top). The orientations for $-90,0,+90$ deg are illustrated at the bottom of Fig. 1. Considerable mathematical simplification in writing equations was gained by designating orientations in this manner.
The longer edges of the rectangular display sheets were parallel to the 90-deg, i.e., vertical, orientation. A large transparent acetate overlay containing a black dot (1.5 $\mathrm{mm}$ diam) near the center covered the display. The 0 , standing at the edge of the table, leaned over to peer down perpendicularly at the display. He was told to "... place the dot at the point where the two lines would intersect if they were extended." Eye movements were not restricted; $O$ was told not to tilt his head to either side. Every 0 judged all 39 displays in an individually randomized order.

\section{Supplementary Experiment 1}

The original experiment was replicated for the subtended angle of $120 \mathrm{deg}$, exercising more stringent control of head tilt and eye-to-display distance. In the main study, $\mathrm{Os}$ were asked, and observed by $E$, not to tilt their heads. Now, a rigid opaque viewing mask with narrow horizontal eye slits was introduced directly above the center of the display so that eye-to-display distance was maintained at $54 \mathrm{~cm}$ and when 0 tilted his head he could not view the display with both eyes. In addition, the field containing the display was changed from a rectangular to a large circular field of $46 \mathrm{~cm}$ diam. The $O$ s were a new sample of 30 students.

\section{RESULTS}

The bidimensional deviations of every judgment were recorded initially in millimeters, using a Cartesian coordinate system with the angle bisector as ordinate and the true vertex at zero. The means of these deviations are listed in Table 1. Computer plots of the raw data provided a scatter diagram for each of the 39 stimulus conditions. (A discussion and an example of such dispersions appear in Weintraub \& Virsu, 1971.) Reducing the data to two-dimensional mean deviations in millimeters in order to treat the results as displacements of a perceived vertex led us to no new insights. Another interpretation of the data can be made in terms of misperceived tilt. It was assumed that each line segment comprising an angle rotates perceptually about its own midpoint to point toward the misperceived vertex (illustrated in Fig. 1, Weintraub \& Virsu, 1971); this assumption will be employed throughout the paper. Note that tilt errors for a pair of lines forming a subtended angle are equal in magnitude but opposite in direction only when $0^{\prime}$ 's judgment lies somewhere along the angle bisector or its extension. Every judgment of the vertex in Cartesian coordinates was transformed into a tilt error for the clockwise line and a tilt error for the counterclockwise line of the pair forming the angle. By averaging the data across 0 s, a mean tilt error in degrees was computed for every line segment of every subtended angle in every orientation, a total of 78 means. The transformation converted a two-dimensional variable into two unidimensional measures. The variability about the mean provides an estimate for the variance in tilt bias associated with a line segment. These data, shown in Fig. 2, became the data to be interpreted.

What of angle misperception? The tilt biases of pairs of line segments forming a subtended angle can be added to give the measures of angle misperception plotted in Fig. 1. An alternate computing procedure for determining angle misestimation is the one employed in the previous study (Weintraub \& Virsu, 1971), converting the mean location of the vertex in millimeters, the data of Table 1 , into angle misestimation. As a consequence of the distribution of $\mathrm{Os}^{\prime}$ ' judgments, a slightly different set of means is thereby obtained: The function for a subtended angle of $120 \mathrm{deg}$ is nearly identical to the one plotted (means were, on the average, .03 deg higher); for the 60-deg subtended angle, all 


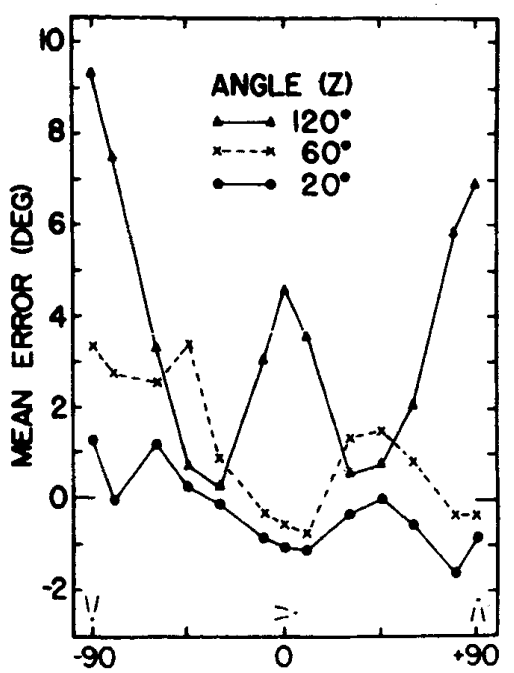

TILT (S) OF BISECTOR (DEG)

Fig. 1. Empirical angle misestimation as a function of subtended angle $(z)$ and orientation of the bisector (z). Positive errors represent overestimation.

points were displaced slightly downward from those shown (an average of .24 deg); for the 20-deg subtended angle, all points were displaced even farther downward (an average of $.46 \mathrm{deg}$ ). In summary, the shapes of the functions show inconsequential changes, but the 20 and 60-des functions are displaced downward if averaging is performed prior to converting to degrees. A positive error denotes an overestimation of the angle, i.e., the judged intersection was too close to the line segment. A subtended angle of $120 \mathrm{deg}$ is overestimated in all orientations, $60 \mathrm{deg}$ is usually overestimated and $20 \mathrm{deg}$ is usually underestimated (contrary to the dictum that acute angles are always overestimated). Superimposed upon the cyclic variations, there was a slope of the functions toward the right, a downward trend in errors as subtended angles proceed from inverted to upright orientations.

The data of primary interest are those associated with individual lines. The tilt-assimilation factor applies to line segments, not to subtended angles. In addition, any representation like that of Fig. 1 obscures the fact that line pairs forming an angle usually show unequal tilt biases, and thus a crucial feature of the experimental outcome is ignored. Figure 2 shows the errors in perceived orientation associated with each line segment. The replication data for the subtended angle of 120 deg are also plotted, and they agree well with the results of the main experiment. It may be assumed that random head tilts, small variations in eye-to-dieplay distance, and the shape of the bordens of a display exert a minor influence upon the outcome of the experiment. There is a clear cyclic component in all six functions consistent with the hypothesis of perceptual tilt of line segments toward the more closely aligned visual axis, either the horizontal or vertical defining $O$ 's visual field. However, the simple plan of removing the cyclic variation mathematically in order to examine the residual as a possible meacure of ande mivestimation was too optimistic. In Fig. 1, there is a downward trend in judgmental error associated with the orientation of the display from inverted to upright. The bias was dubbed the "elevator factor" because $O$ emed to place the dot representing the vertex too high in his visual field. For example, with bisector at $-90 \mathrm{deg}$, if the 0 places the dot too high, then he will place it closer to the line segments, giving an additional component of overestimation to the subtended angle. With the bisector at

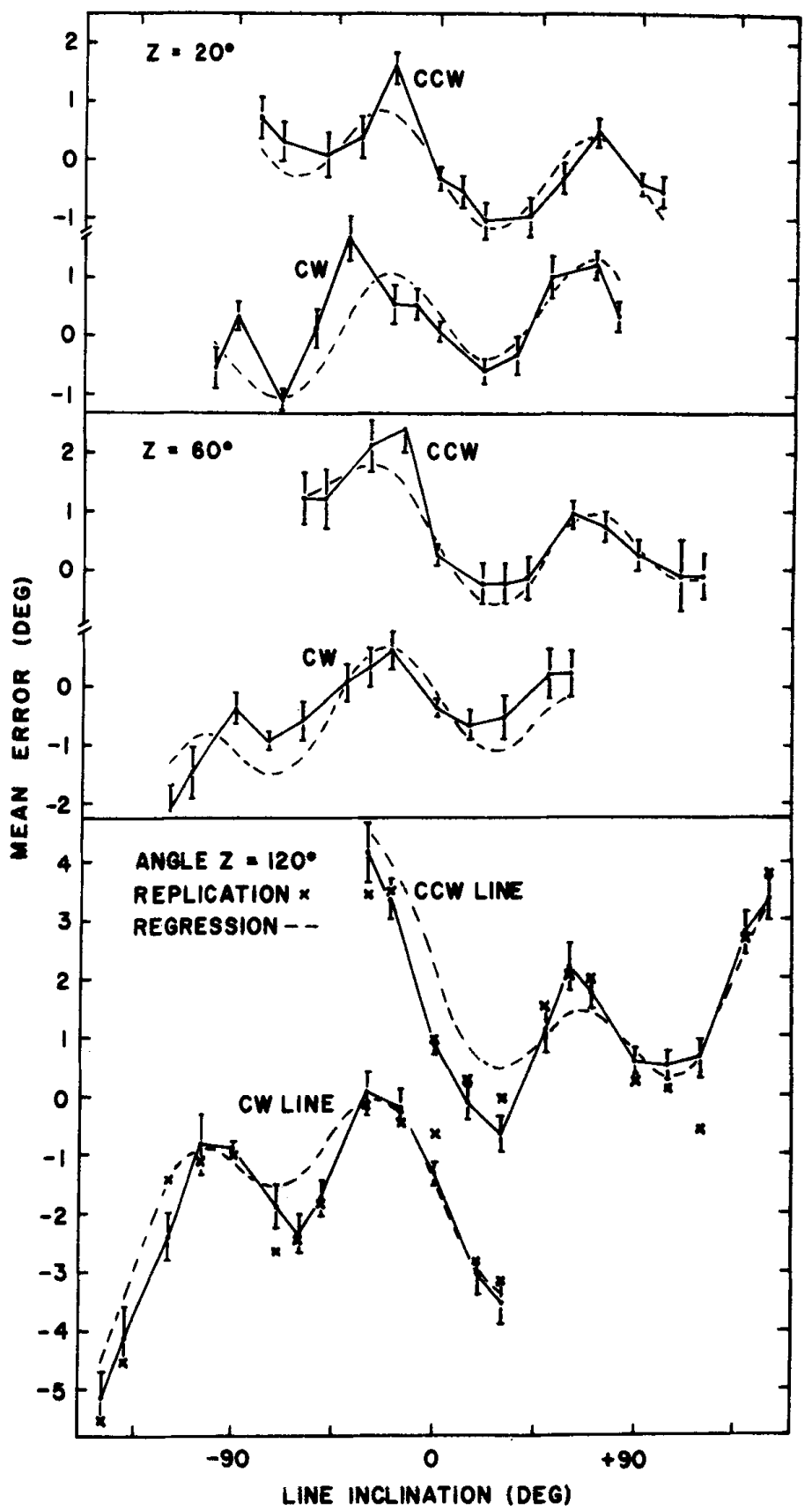

Fig. 2. Frrors in the perceived inclination of individual line segments. Counterclockwise errors are designated as positive. 
+90 deg, a dot too high will put it farther from the line segments, giving an underestimation component. When the bisector is not oriented vertically (all orientations except $\pm 90 \mathrm{deg}$ ), placing the dot too high also moves it off the angle bisector. Proposing independent status for the bias was sparked by the observation that a similar effect had occurred in another experiment. When the Poggendorff display was inverted, an increased error was found (Weintraub \& Krantz, 1971). Before we decided to incorporate the apparently systematic effect into our schema, the bias was investigated in its own right.

\section{Evidence Concerning \\ the Elevator Factor}

An empirical calculation of the elevator factor is possible. For a given subtended angle, two orientations that deviate equally but in opposite directions from horizontal (i.e., from zero bisector tilt) can be treated as a matched pair of subtended angles; a subtended angle of $20 \mathrm{deg}$ at +60 - and -60 -deg bisector tilt form such a pair. For each orientation of the pair, the mean vertical (with respect to the 0 ) component of the error in millimeters can be computed. The question asked is, disregarding lateral error, how many millimeters too high or low was the average judgment for that orientation? The elevator factor should be the same in magnitude and sign for each member of the matched pair. However, the contribution of other factors to the vertical error relative to $O$ ought to be equal but of opposite sign for each member of the pair. Thus, the reversal of sign will occur for tilt-assimilation errors. Also, other systematic biases like misperception of subtended angle should occur with respect, not to $O$, but to the displays, one of which is inverted relative to the other, providing for reversed signs. By adding the vertical error components from matched pairs of subtended angles and dividing the result by 2 , an elevator factor was extracted. (Addition cancels effects with opposite sign and doubles the size of the elevator factor.) One can visualize the process most easily for an upright-inverted ( $\pm 90 \mathrm{deg})$ pair, but the same reasoning applies to all matched pairs.

The computations revealed that the elevator factor was not a constant. It was nearly zero when the bisector of an angle was horizontal, and as an angle approached either an upright or inverted position, there was an increasing upward bias to the settings. The slope of the functions in Figs. 1 and 2 is a manifestation of the effect. Since the experimental procedure permitted two-dimensional errors, it was possible to calculate the standard

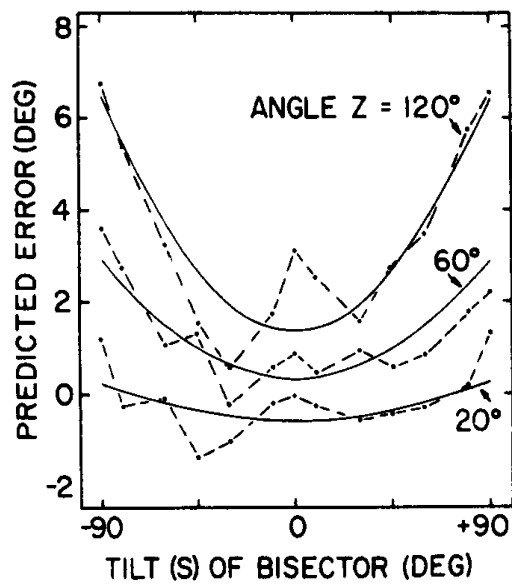

Fig. 3. Empirical angle misestimation after removing components that are independent of the subtended angle (z). Positive errors represent overestimation. The smooth functions were derived from the model.

deviation of the vertical component of every mean setting. From a minimum at the horizontal orientation of the angle, the vertical component of the standard deviation increased to a maximum as the bisector reached either an upright or inverted position. The empirical bias values in millimeters were correlated with the equivalent vertical component of the standard deviation in millimeters. The product-moment correlation was +.86 , an outcome highly significant statistically. The correlation indicates that the elevator factor for horizontal displays is slight and that there is only small vertical variability in these vertex settings. The elevator factor increases to a maximum as the bisector approaches vertical where the vertical variability is large. One must be cautious in inferring cause and effect from a correlation, but it is tempting to conclude that the elevator factor is constrained to operate in direct proportion to the uncertainty in the location of the vertex vertically. The existence of such a correlation raises our confidence that the elevator factor is worthy of status as an entity.

\section{Supplementary Experiment 2}

An experiment was designed to investigate the possibility that the upward bias is methodological, perhaps in the same class with the known psychophysical errors of anticipation and habituation. A variant of the method of constant stimuli, the group version of the up-and-down method, was employed with 48 new Os. (See Weintraub \& Krantz, 1971, for details, which include statistical testing.) Only six displays were judged by each $O$, the upright and inverted orientations of the three angles of the main experiment. Each 0 observed the dot in a fixed position along the bisector of the display and was required to state whether it was placed too low or too high to mark the vertex of the angle.

For subtended angles of 20,60 , and $120 \mathrm{deg}$, the differences in errors between inverted and upright settings were $16.66,6.53$, and $2.78 \mathrm{~mm}$, respectively. The differences indicate the usual upward bias, and three $z$ tests of these differences were all statistically significant (in each instance at least $\mathrm{p}<.05$, two-tailed). The result indicates that the elevator factor is not tied to a particular psychophysical method but may well be a perceptual factor. (No left-right lateral bias has been found. The left side of the visual field was selected in these experiments in order to compare the data with Weintraub \& Virsu, 1971, where the right side was used.)

Regression Analysis

The following four-factor additive regression model was devised for analyzing the results:

$$
y=b_{1} \sin 4 x+b_{2} s+b_{3} z s^{2}+b_{z},
$$

where $\mathrm{y}$ is the tilt error in degrees of each individual line segment; $b_{1}, b_{2}$, $b_{3}, b_{z}$ are parameters; $x$, the angular deviation of each individual line segment from the horizontal; $z$, the angle subtended between line segments; and $s$, the orientation of the bisector of angle $z$, that is, $s=x-1 / 2 z$ for the counterclockwise line of a pair and $s=x+1 / 22$ for the corresponding clockwise line.

Term $b_{1} \sin 4 x$ accounts for perceptual tilt (assimilation) of each single line toward the more closely aligned visual axis, horizontal or vertical. It has been used as an approximation for single-line tilt errors before (Bouma \& Andriessen, 1968; Virsu, 1971).

The approximation term for the elevator factor is $b_{2} s$. The upward bias, determined algebraically in millimeters, was converted to an equivalent tilt error in degrees for each line segment of each subtended angle in each orientation. The formula for conversion to degrees is a mathematically messy trigonometric function of the upward millimeter bias. Whenever a line segment is positioned vertically $(x= \pm 90 \mathrm{deg})$, even a large elevator factor, because it also operates vertically, causes no tilt error. Or, when the bisector (s) of the subtended angle is horizontal, the elevator factor was near zero regardless of the inclination $(x)$ of a line segment, which depends upon the particular subtended angle (z). As stated previously, the upward bias in millimeters increased from zero as the 
orientation of the subtended angle deviated from horizontal (i.e., from $s=0$ ), and a simple but effective approximation was a linear $b_{2} s$ term with $b_{2}$ negative for any counterclockwise line and positive for any clockwise line of a pair. ${ }^{1}$ The functions in Fig. 2 show the slope trends superimposed upon other systematic variations.

Imagine that suitable values for coefficients $b_{1}$ and $b_{2}$ are applied to the first two terms of the model, that these components are then subtracted from the six curves of Fig. 2, and that, for lines forming a subtended angle in a given orientation, the remaining components of judgmental error are summed for each pair and plotted. The outcome can be considered as removal of the tilt-assimilation factor and the elevator factor from the angle-misestimation data of Fig. 1. A neat, easily interpretable "corrected" Fig. 1 would consist of three horizontal lines, meaning that the remaining error is independent of the orientation of the subtended angle. The distance of each horizontal function from a mean tilt error of zero would achieve the goal, angle misestimation as a function of subtended angle. However, the actual outcome was a nested set of functions opening upward, roughly parabolic in shape, symmetrical about $s=0$. Figure 3 illustrates such corrected data; the $b_{1}$ and $b_{2}$ coefficients were derived, in this instance, from the least-squares best-fit solution for the entire four-term regression model in its final formulation, as written above. The remaining error had an additional important property: It tended to be distributed equally between each line segment of a pair. Therefore, the four-term model, which is intended to account for the tilt error of an individual line segment, was designed to predict half the remaining error.

Term $b_{z}$ encompasses the residual component of angle misperception that is independent of the orientation of the angle. Without assuming any function to explain the differences among angles, $b_{2}$ must be estimated separately for each angle $z$.

The other residual component varies with the orientation of the angle so that the differences between the two error functions for the pair of line segments forming an angle become larger when the bisector of the angle approaches a vertical position, i.e., there is more overestimation (or less underestimation) of the angle as its bisector approaches vertical. The tendency is stronger, the larger the $s u b t e n d e d$ angle $z$, and an approximation for this error component is directly proportional to angle $z$ and to the square of the deviation of the bisector from the horizontal. The expression $b_{3} z s^{2}$ is a simplification, but it is adequate for the range of subtended angles in the present experiment.

A least-squares solution of the parameter values of the regression model was obtained through a series of successive linear regression solutions. In all, six estimates are required, one value each for $b_{1}, b_{2}, b_{3}$, and $a b_{2}$ for each angle $z$. All the mean data were utilized in the estimation of every parameter. The resulting parameter values were $b_{1}=-.8223$, $b_{2}=\mp .006321, b_{3}= \pm .000002622$, $b_{20 \mathrm{deg}}=\mp .2911, \mathrm{~b}_{60} \mathrm{deg}= \pm .1658$, and $b_{120} \mathrm{deg}= \pm .6829$. The upper signs refer to the more counterclockwise line and the lower signs to the more clockwise line of each pair. The standard error of the estimate was .5159. The smooth dotted curves in Fig. 2 show the fit of the regression model to the results. The fit is reasonable (multiple correlation .945), and $89 \%$ of the variance of the mean errors is explained by the regression model. Each of the four components yielded a statistically highly significant contribution to the explanation of the total variance.

Each term of the regression model can be employed alone by dropping all other terms and using a least-squares best-fit criterion to determine the remaining coefficient. The tilt-assimilation term containing $b_{1}$ explains $59 \%$ of the variance of the mean tilt errors when it is the sole term, the elevator factor (with $b_{2}$ ) $34 \%$, the orientation-dependent component of angle misperception $\left(b_{3}\right) 66 \%$. The $b_{z}$ term, actually three values, one for each subtended angle, nonetheless accounts for only $32 \%$ of the variance. Since the largest proportion of variance is explained by the orientation-dependent component of angle misperception, it is meaningless to refer to the misperception of an angle without reference to the orientation of the angle in the visual field, even after removing single-line effects.

The data of Fig. 1 stand as empirical angle misperception. However, the four-factor model implies that two of the components leading to errors in misperceiving a vertex, tilt of individual line segments toward a cardinal visual axis, and the elevator factor, are independent of the subtended angle itself. Therefore, by employing only the last two terms of the model, which are dependent upon the angle subtended, a smoothed prediction of "uncontaminated" angle misestimation can be obtained. The point at which each parabolic function has its minimum occurs when the angle bisector is horizontal $(s=0)$, and the error at the minimum is twice the $b_{z}$ term for any angle $z$. The smooth functions of Fig. 3, then, represent a best prediction of purified angle misperception for subtended angles of 20,60 , and $120 \mathrm{deg}$ for angles formed by nonintersecting lines when the defining operation is to determine the apparent vertex. The empirical data of Fig. 3 correspond to residual tilt errors after removing the components corresponding to the first two terms in the model, and these data contain all the nonsystematic (random) error as well as systematic error introduced by the inappropriateness of the model. The first two terms of the model do operate as advertised, accounting for cyclic variations and sloping functions.

\section{DISCUSSION}

The model evolved logically. The $b$, and $b_{2}$ terms were planned; the $b_{2}$ term was added after corollary evidence was gathered; the $b_{3}$ parabolic term was then selected to approximate the remaining systematic variation. Among the infinity of alternative descriptions, many are reasonable. For examples, greater tilt assimilation may obtain toward one of the visual axes than toward the other, or size of subtended angle may be assumed to interact with tilt assimilation. We were unable to devise a formulation that captured the essence of the tilt-assimilation and elevator factors such that only one additional factor, which could be made to be independent of angle orientation, ascribable to angle misperception, was necessary. The stumbling block for an economical mathematical description is that the logically different factors are not easily specified in the same units or by the same parameters. The regression model serves as a useful analytic description of the results. Our hope is that the first two terms represent phenomena that exist beyond the confines of the vertex-location paradigm. The model has generality at least beyond the present experiment. In the earlier study (Weintraub \& Virsu, 1971), the subtended angle was varied systematically. In one experiment, the bisector of the angle remained vertical and in the other the left line segment was held at vertical while the angle varied over a large range of values. Dropping the unknown component $b_{z}$ from the model, the model explains $71.6 \%$ of the variance of mean errors of those experiments, using only the three values $b_{1}, b_{2}$, and $b_{3}$ retained from the present experiment. The result implies good stability of the parameter values over a large variation in display orientations and angles between the line segments.

There are two approaches to the study of angle misestimation. One is 
indirect, like using judgments of the apparent vertex to infer that angle misestimation has occurred. Often, the motive for this type of study is to explain a visual illusion-the Poggendorff serves as a prime example-or to support hypotheses about interactions among the neural representations of contours.

Corrigan (1970) conducted similar experiments with a dot-setting technique for finding the vertex of converging line segments. The movement of the dot was restricted to the imaginary bisector of the subtended angle or its extension so that no lateral errors were permitted. Since Os make very few lateral errors when the angle bisector is oriented vertically, Corrigan's one-dimensional vertical settings should provide data equivalent to two-dimensional settings, and they do. Figure 3A of Weintraub and Virsu (1971), which is plotted in millimeters, serves as a tolerable substitute for Corrigan's own graph. Corrigan concluded from displays in three orientations that angles generally tend to be overestimated, and that there is tilt assimilation of lines toward the vertical axis only. We feel confident that his conclusions would have more closely matched our own if two-dimensional judgments had been permitted and/or more orientations had been assayed. A problem must arise for the $O$ when no setting can quite match his subjective vertex. (Does he minimize the discrepancy?) Information about the subjective vertex is undoubtedly lost.

Bouma and Andriessen (1970) have also employed a dot-setting procedure. However, they empirically corrected their data on two lines (subtended angle) for single-line effects by subtracting single-line "control" data from the two-line data. The validity of the subtraction procedure rests upon the implicit assumption that single-line effects are additive and involve no interactive components. The four-factor model lends support to this assumption. Bouma and Andriessen (1968) have found tilt assimilation toward both horizontal and vertical for single lines.

The second approach treats angle misperception as a performance problem to be confronted directly by requiring Os to produce an angle that matches the standard, or to make a verbal estimate in degrees. Two recent studies used the production method (Beery, 1968; MacLean \& Stacey, 1971), but the comparison matched the standard in orientation. The four-factor model predicts zero judgmental error because all effects are acting equally on each display. That is, in a null-match experiment, where both standard and comparison are visible simultaneously and oriented similarly, they should be perceived as identical. Location in the visual field, attentional differences, etc., may be operating, for which the model was not designed. For predictions of other than zero from the model, it is necessary for the standard and comparison angles to be presented in different orientations. Fisher (1969) and MacLean and Stacey (1971) also employed verbal estimation, about which the model might make defensible predictions. The startling finding is that either direct method yields results diametrically opposed to those of the vertex-location technique. MacLean and Stacey confirm Fisher in concluding that an angle whose bisector is oriented vertically ( $s= \pm 90 \mathrm{deg}$ ) is judged as smaller than the same angle oriented horizontally (s=0 deg); Fig. 1 shows just the opposite. Furthermore, the Weintraub-Virsu (1971, Fig. 3A) data for displays oriented vertically $(s=90$ deg $)$ show that underestimation occurs for acute angles less than $60 \mathrm{deg}$; all other angles a re overestimated. The MacLean-Stacey (1971, Fig. 3) data show overestimation for the smaller acute angles and underestimation elsewhere. Beery (1968) concluded that, rather than tilt assimilation, there is tilt repulsion away from horizontal and vertical.

Besides the nature of O's task, there are other methodological differences. The new data are based upon displays with nonintersecting lines forming the angle. (There is reason to believe that intersecting lines will not produce equivalent perceptual outcomes. See Weintraub and Virsu, 1971, p. 8.) Also, the distances between the line segments were large, up to 12.7 deg of visual angle. Therefore, the interaction effects between the line segments are not easily explained on the basis of inhibitory interactions between contour or orientation detectors (see Ganz, 1966; Blakemore, Carpenter, \& Georgeson, 1970; Bouma \& Andriessen, 1970) because strong inhibitory neural influences cannot be expected over such large distances. Some other features of the results, such as the overestimation of the 120-deg angles in all orientations, are also difficult to understand as inhibitory interactions.

In summary, as the joint result of the earlier and present experiments, it is possible to conclude that, when the vertex of two converging, relatively long line segments was estimated, the judgments were consistent with the following generalizations:

(1) A line segment appears perceptually tilted toward the more closely aligned axis either the horizontal or vertical of the visual field.
(2) There is a tendency for $O$ to estimate the apparent vertex too high in his visual field.

(3) An interaction effect between line segments exists suggesting misperception of the angle subtended. The interaction depends strongly upon the orientation of the angle.

(4) The overall outcome (Fig. 1) is that $20 \mathrm{deg}$ is underestimated in most orientations, $60 \mathrm{deg}$ is usually overestimated, $120 \mathrm{deg}$ is always overestimated.

The two-dimensional vertex-location technique produces reliable data, possesses methodological advantages, has face validity as a means of assessing angle misperception. The fact that it produces discrepant data raises questions concerning all techniques. An encouraging facet of the evidence is that, although disparate methods strongly disagree as to the direction of errors, they tend to implicate the same explanatory variables. Since we already hold a strong bias that angle misperception is multiply caused, additional causative agents linked to different procedures would be not at all surprising.

In any event, a four-term mathematical statement presents a specific target and an open invitation to all parties to commence firing. Given the bumps and grinds in the data, four factors may prove highly constraining when confronted with new evidence. In our present optimism, we believe that, when the smoke clears, the model will be neither badly mutilated nor mortally wounded. Whatever the outcome, it looks like an exciting shoot-out.

\section{REFERENCES}

BEERY, K. E. Estimation of angles. Perceptual \& Motor Skills, 1968, 26, 11-14.

BLAKEMORE, C. CARPENTER, R. H. S. \& GEORGESON, M. A. Lateral inhibition between orientation detectors in the human visual system. Nature, 1970, 228, 37-39.

BOUMA, H., \& ANDRIESSEN, J. J. Perceived orientation of isolated line segments. Vision Research 1968, 8, 493-507.

BOUMA, H., \& ANDRIESSEN, J. J. Induced changes in the perceived orientation of line segments. Vision Research, 1970, 10, 333-349.

BURNS, B. D., \& PRITCHARD, R. Geometrical illusions and the response of neurones in the cat's visual cortex to angle patterns. Journal of Physiology, $1971,213,599-616$.

CHIANG, C. A new theory to explain geometrical illusions produced by crossing lines. Perception \& Psychophysics, 1968, 3, 174-176.

CORRIGAN, B. Constant error in the perceptual extrapolation of straight lines. Paper presented at the meeting of the Western Psychological Association, Los Angeles, April 1970.

FISHER, G. H. An experimental study of angular subtension. Quarterly Journal of Experimental Psychology, 1969, 21, 356-366.

GANZ, L. Mechanism of the figural 
aftereffect. Psychological Review, 1966, 73, 128-150.

MacLEAN, I. E., \& STACEY, B. G. Judgment of angle size. Perception \& Psychophysics, 1971, 9, 499-504.

O B O N I T. Experimentelle Untersuchungen über den Aufbau des Untersuchungen über den Aufbau des
Sehraumes. Archiv der gesamten Psychologie, 1931, 82, 308-328.

VIRSU, $V$. Tendencies to eye movement, and misperception of curvature, direction, and length. Perception \& Psychophysics, 1971, 9, 65-72.

WEINTRAUB, D. J., \& VIRSU, V. The misperception of angles: Estimating the vertex of converging line segments. Perception \& Psychophysics, 1971, 9, 5-8.

WEINTRAUB, D. J. \& KRANTZ, D. H

The Poggendorff illusion: Amputations, rotations, and other perturbations Perception \& Psychophysics, 1971, 10, 257-264.

NOTE

1. Roughly speaking, as the orientation of the bisector proceeds from -90 through 0 to +90 deg for the counterclock wise line of a pair, the tilt error component resulting from the elevator factor proceeds from positive (counterclockwise error) through zero to nezative (clockwise error). The clockwise line, conversely, has a positive slope. (The reader who sketched out the shape of these functions would find a hump in each that is ignored by the linear approximation.)

(Received for publication November 8 , 1971. 